\title{
Réécritures bibliques contestataires dans El infinito en la palma de la mano de Gioconda Belli et Sara de Sergio Ramírez
}

Nathalie Besse

\section{(2) OpenEdition} Journals

Édition électronique

URL : https://journals.openedition.org/cher/2269

DOI : $10.4000 /$ cher.2269

ISSN : 2803-5992

Éditeur

Presses universitaires de Strasbourg

\section{Édition imprimée}

Date de publication : 29 mai 2018

Pagination : 109-120

ISBN : 979-10-344-0019-5

ISSN : 1968-035X

Référence électronique

Nathalie Besse, « Réécritures bibliques contestataires dans El infinito en la palma de la mano de Gioconda Belli et Sara de Sergio Ramírez », reCHERches [En ligne], 20 | 2018, mis en ligne le 01 décembre 2021, consulté le 02 décembre 2021. URL : http://journals.openedition.org/cher/2269 ; DOI : https://doi.org/10.4000/cher.2269

\section{cc) (1) $\$($ )}

$\mathrm{Ce}(\mathrm{tte})$ œuvre est mise à disposition selon les termes de la Licence Creative Commons Attribution -

Pas d'Utilisation Commerciale - Partage dans les Mêmes Conditions 4.0 International. 


\title{
Réécritures bibliques contestataires dans El infinito en la palma de la mano de Gioconda Belli et Sara de Sergio Ramírez
}

\author{
Nathalie Besse ${ }^{1}$
}

\begin{abstract}
$\mathrm{L}$ es deux principaux romanciers nicaraguayens, internationalement reconnus, ont réécrit chacun un texte biblique: Gioconda Belli propose une réécriture de l'épisode génésiaque de la Chute, fondateur du genre humain, dans El infinito en la palma de la mano (2008), prix Biblioteca Breve Seix Barral; Sergio Ramírez réinvente l'histoire de Sarah et Abraham dans Sara (2015). Ce transfert d'un texte saint au roman, à un contexte générique différent, et la perspective d'auteurs que bien des siècles séparent du texte originel, offrent un regard nouveau, empreint de questions aussi actuelles qu'intemporelles.

Selon quelles modalités discursives s'expriment ces réécritures? Quels en sont les jeux et enjeux? Si Gioconda Belli et Sergio Ramírez réécrivent chacun un passage de la Genèse, texte des origines, comme tel fondateur, et lié au fondement de l'être, ces textes bibliques sont romancés non sans intentions "politiques": la réécriture opère ici de façon subversive, exprimant la contestation d'un pouvoir, androcentrique ou absolu, au moyen d'un personnage féminin à même de métaphoriser l'exclusion ou la domination de l'autre.

D’une Ève insoumise dans les écrits féminins et féministes de Gioconda Belli qui s'impliqua en son temps dans la révolution sandiniste, à une Sarah indocile dans l'écriture masculine d'un romancier autrefois vice-président du gouvernement révolutionnaire et depuis lors défiant face aux maux du pouvoir, ces réécritures du texte primordial passées au crible de l'Histoire adaptent des figures traditionnelles pour offrir à l'homme d'aujourd'hui une vision critique de son époque. Elles défendent des droits aussi fondamentaux que la liberté qui se manifeste ici par la pensée ou le rire entre autres exemples de désobéissance.
\end{abstract}

1 Maître de conférences HDR en espagnol, Université de Strasbourg. 
Il s'agira d'abord de montrer en quoi ces réécritures du texte des origines s'inscrivent dans une perspective humaniste, puis comment elles interpellent le pouvoir, se donnant à lire ainsi comme des réécritures contestataires, et à leur manière politiques.

\section{Réécritures humanistes: origines des textes et textes des origines}

"Tout texte est absorption et transformation d'un autre texte», a pu écrire Julia Kristeva en 1966 dans Semeiotikè où elle définit le terme d' "intertextualité » (85); la réécriture n'est-elle pas, au même titre que l'intertextualité, la condition de tout texte?, toute écriture s'avérant dès lors réécriture en soi. Gioconda Belli et Sergio Ramírez se sont plus d'une fois inspirés de textes préalables mais dans El infinito en la palma de la mano et Sara, chacun réécrit délibérément un texte "universel" dans son intégralité séquentielle.

Entendons-nous ici sur le terme «réécriture»: Gioconda Belli explique, dans la «Note de l'auteur» qui précède le roman, l'origine de «la idea de reconstruir el drama de Adán y Eva», depuis l'éveil de la conscience humaine jusqu'au crime de Caïn (11). S'inspirant de versions apocryphes de l'Ancien et du Nouveau Testaments écrites dans l'Antiquité, qu'elle a trouvées de façon fortuite dans une bibliothèque, elle affirme: "Ésta es pues una ficción basada en las muchas ficciones, interpretaciones y reinterpretaciones que alrededor de nuestro origen ha tejido la humanidad desde tiempos inmemoriales» $(13)^{2}$. Reconstruction, (ré)interprétation, fiction, la réécriture est explicitement assumée ici.

Sergio Ramírez, lorsqu'il étoffe les quelques versets bibliques concernant Sarah et Abraham pour en faire un roman de quelque 250 pages, prétend «réécrire» ou "recréer», non pas un texte sacré ou dogmatique comme il s'en explique dans plusieurs entrevues accordées à l'occasion de la parution de l'ouvrage, mais une «histoire» - la fiction encore:

[...] una historia que pudiera reescribir, porque la Biblia está llena de muchas historias interesantes. Sobre todo la parte del Antiguo Testamento. El contexto de la historia y lo poco que la Biblia habla de ellos, a pesar de que son muy famosos, me atrapó. [...] vi que le podía sacar mucho partido como novelista, no como teólogo, ni como historiador. Sólo recreo el texto como lo han hecho escritores (Sevilla Bolaños 2015)³.

Dans cette fiction il s'intéresse à la première épouse de patriarche, mère du peuple d'Israël, une femme pourtant stérile et qui, lorsqu'on assure à son mari Abraham qu'il sera l'ancêtre d'une multitude, ne peut s'empêcher de rire intérieurement (Genèse, 18,12 ou 21,6) comme le rappelle une première épigraphe qui place tout le roman sous le motif principiel du rire dont nous analyserons plus loin les enjeux. Car ce n'est pas sans raison que l'auteur revisite sous cet angle une figure que l'apôtre Paul présente, dans l' «Épître aux Hébreux», comme un exemple de

2 C'est nous qui soulignons.

3 C'est nous qui soulignons. 
femme soumise et un modèle de foi : ici contestataire et incrédule, cette Sara bien différente de celle que connaissent les exégètes, entre dans un rapport de force avec Dieu, à l'instar de l'Ève que conçoit Gioconda Belli.

Mais c'est d'abord comme possibles représentations de tout un chacun, que ces personnages bibliques, en cela hautement symboliques - sinon paradigmatiques dans le roman de Gioconda Belli -, ont été choisis par des auteurs visiblement soucieux de rendre compte de l'humaine condition. Ainsi Sergio Ramírez, qui rappelle que la Bible n'a suscité ici son intérêt qu'en tant que «source inépuisable d'histoires humaines », apprécie ses silences qui offrent beaucoup d'espace pour lire entre les lignes, notamment dans l'Ancien Testament: "tienen que ver con las luchas por el poder, con los celos, con los triángulos amorosos. Y con seres llenos de contradicciones y debilidades» (Mendoza 2015).

Gioconda Belli également universalise l'histoire du premier couple lorsqu'elle achève sa «Note de l'auteur» par: «Es, en su asombro y desconcierto, la historia de cada uno de nosotros» (13); aussi bien, Adam signifie-t-il en hébreu «homme» ou «humanité». L'auteur s'interroge sur le premier homme et la première femme pour mieux comprendre l'humanité d'aujourd'hui, son inhumanité aussi lorsqu'elle montre un homme en proie à ses contradictions ou à des questions profondes, oscillant entre Bien et Mal.

Les lignes de El infinito en la palma de la mano rappellent, à cet égard, des conceptions existentialistes et ce dès l'épisode génésiaque de la transgression puisque la liberté d'Adam et Ève de goûter ou non au fruit interdit - liberté explicitement énoncée dans le roman: "poseen la libertad de decidir lo que quieren. Son libres de comer o no comer de este árbol» (28) -, les met face à une immense responsabilité qui est au cœur d'une réflexion sartrienne bien connue. Seul face à lui-même et face à son existence, responsable de tout excepté de sa responsabilité même, et portant sur ses épaules le poids du monde, puisqu'en choisissant entre le savoir ou l'éternité il engage l'humanité entière, Adam devient mortel tout en perpétuant l'humanité même, et avec elle l'Histoire.

Et ce savoir «causa inquietud, inconformidad. Uno cesa de aceptar las cosas como son y trata de cambiarlas» (27) selon un Serpent célèbre instruisant Ève; c'est là l'essence et l'enjeu même de ces réécritures fictionnelles humanistes mais également contestataires à leur façon, qui questionnent versions officielles et pouvoir(s), mettant en scène des femmes qui veulent savoir et bouleversent ainsi l'ordre établi, car si le savoir est pouvoir il est aussi contre-pouvoir.

\section{Réécritures contestataires: une femme face au pouvoir absolu}

Si Ève et Sara sont présentées à la troisième personne au même titre qu'Adam et Abraham, chacune n'en demeure pas moins le personnage principal: c'est depuis la perspective du personnage féminin que s'exprime le narrateur omniscient qui nous fait part de leurs pensées et ressentis, et l'on comprend bien, lors des confrontations dialoguées avec l'homme et avec Dieu, que les auteurs 
animent de leur propre point de vue ce personnage frondeur. Car c'est dans cette révolte, nous l'avons dit, que résident la nouveauté et la subversion de ces réécritures romanesques.

Dans El infinito en la palma de la mano, une relecture inattendue de la Genèse amène une Ėve contestataire, et à travers elle une romancière dont la biographie témoigne assez de la révolte, à accuser le créateur (ici appelé Elokim) d'avoir programmé la faute, et à le désacraliser en lui prêtant des faiblesses humaines.

Concernant le premier point, Ėve n'a de cesse d'incriminer Dieu, coupable d'avoir condamné ses créatures à la faute, un déterminisme que ne renie pas le Serpent: libres et conditionnés tout à la fois, Adam et Ève décident en obéissant à un ordre supérieur. Le discours exprime de façon patente cette ambiguïté en juxtaposant liberté humaine et volonté divine, de même qu'il rend compte de la fatalité au moyen de "para (que)» dont plusieurs occurrences émaillent cette réécriture irrévérente, par exemple: «Harían la Historia para la que habían sido creados» (39).

Nul péché d'orgueil humain dans cette transgression qui obéit à un dessein divin, et donc nulle culpabilité de la femme: Gioconda Belli, fidèle à l'impertinence que connaissent ses lecteurs, accuse sans ambages le créateur qui espérait en outre se déresponsabiliser. Car cet Elokim revu et corrigé par les soins querelleurs de Gioconda Belli, qui brosse ici le portrait anthropomorphiste d'un Dieu à l'image de l'homme, ne brille pas par son courage, deuxième point annoncé supra. Face à cet Elokim tout-puissant et paradoxalement inquiet, se dresse la femme, celle qui ose savoir - parce qu'elle veut être tandis que l'homme se contente de vivre? - dans une subversion des Écritures ou une réécriture du discours biblique dont les "amendements" ne sont pas sans rappeler le discours bellien de libération de la femme.

À la différence d'Adam qui respecte la loi divine, Ėve veut savoir et comprendre, elle questionne et cherche, sentant le besoin permanent d'“autre chose", s'intéressant non pas à l'acquis mais à ce qui manque - comme si déjà au Paradis elle se sentait incomplète. Face à un Adam qui se satisfait de la connaissance, Ėve paraît préférer la pensée, d'où son indocilité, ou cette «intention» qui l'anime selon Adam (22), et cette tentation qui ici semble représenter en définitive la rançon de l'intelligence. Femme forte, à défaut d'être puissante, comme toutes les protagonistes de Gioconda Belli dont c'est là le vrai pouvoir, elle s'oppose à Elokim en amenant l'homme à la transgression ou en réprouvant ouvertement le créateur - «Eres cruel. [...] No me digas que no lo planeaste» - ou encore en exigeant sur le mode impératif qu'il restitue la lumière (72-73).

Autre schème récurrent sous la plume de Gioconda Belli: le savoir passe par un corps idéalisé, instrument de perception et de connaissance du monde. Lorsqu'il n'est pas lié à la transgression, le corps, porteur de symboles et souvent chargé d'enjeux, révèle, dans ce texte à sa façon féministe et non exempt de manichéisme, un antagonisme femme-homme épousant l'antagonisme 
Bien-Mal ainsi qu'en témoignent des dialogues associant la femme à la vie et l'homme à la mort:

-Yo di vida, Adán. Él que empezó a matar fuiste tú.

- Para sobrevivir.

- No te culpo pero una vez que aceptamos que había que matar para sobrevivir permitimos que la necesidad dominara nuestra conciencia, admitimos la crueldad (224).

Nécessité versus idéal? Dans une sorte de duel verbal, l'obligation exprime, avec une symétrie notable, ce désaccord: "Tendremos que matar» affirme Adam, à quoi Ève répond: «No debes matar. Me lo dice todo el cuerpo»; mais le devoir pour Adam consiste dans la survie plus que dans l'éthique défendue “viscéralement" par Ève: «Sé que debemos comer» (109-112).

Dans Sara, le personnage féminin se dresse à nouveau contre un créateur ici dénommé «el Niño» ou "el Mago», au moyen de la contestation franche et de la mise en doute de la parole divine comme dans le roman précédent, mais également au moyen du rire qui, dès l'épigraphe inaugurale du roman, donne sa tonalité et une certaine intention au récit, ce rire que l'auteur associe à une forme subtile d'intelligence: "una mujer inteligente a la que le prohíben reírse del mundo autoritario pero que se ríe; que defiende la vida de su hijo Isaac de los designios divinos del Mago; que busca ser libre dentro de sí misma y hacia afuera; y que siempre está reclamando un lugar justo en el mundo» (Agüero 2015).

On le voit, la contestation du pouvoir est là encore à l'œuvre: la femme revendique une liberté d'être, ou s'affirme comme un être de liberté. L'auteur a d'ailleurs choisi une femme car c'est d'une part un personnage soumis au système patriarcal, un personnage exclu (Sarah apparaît, dans le texte biblique, derrière le rideau) qui, dans cette réécriture audacieuse, va entrer en conflit avec l'autorité masculine, plus précisément avec la figure majeure du créateur; d'autre part, la femme est censée exprimer ses émotions plus que l'homme, de là cette femme qui rit face à ce qui semble être un mensonge, fût-il l'émanation du pouvoir suprême, tandis qu'Abraham, comme l'Adam de Gioconda Belli, obéit aux règles sans grands dilemmes intérieurs.

C’est précisément cette relation conflictuelle qui intéresse au premier chef l'auteur lorsqu'il choisit de réécrire le personnage biblique de Sarah : «la relación que ella tiene con Dios. Siempre me pareció que es una relación conflictiva y para una buena historia se necesita un buen conflicto. Tengo en frente un doble conflicto: Sara frente a Dios y Sara frente a su esposo Abraham » (Sevilla Bolaños 2015). L'auteur se défie, dans la même entrevue, d'en faire une féministe, mais entend lui restituer un protagonisme dans une société patriarcale faite de relations verticales.

Nous avons dit que l'expression de cette contestation passe, comme toujours dans les romans de Sergio Ramírez, par le rire qui représente une première distance d'avec le pouvoir, et peut signifier l'incrédulité ou le mépris: "No fue 
ninguna carcajada [...] sino una especie de graznido despectivo, que mostraba incredulidad y desprecio. La risa del desdén» (37).

Ce rire irrite le Tout-Puissant: «el que se enoja ante la risa de Sara es el Mago mismo» (43) et suscite les remontrances des messagers, par où l'on voit comment le rire s'oppose au pouvoir, et le pouvoir au rire, ce que l'auteur affirme avec une concision notable: "el poder no conoce el humor", "no le gusta que lo desnuden $»^{4}$. Outre qu'il déstabilise ou fragilise le pouvoir, le rire le contraint et peut inverser le rapport de force: «era la primera vez que el Mago se dirigía a ella [...] ¿No era aquello un triunfo? Aunque fuera a costa de su cólera, lo había doblegado. De algo sirve la risa, se dijo, y volvió a reírse por lo bajo, cuidando esta vez que nadie la oyera» (45).

Nous avons là une légitimation du rire qui apparaît non seulement comme une réponse distancée et critique face au pouvoir, mais également comme un véritable pouvoir en soi, à même de transformer le rapport de force; rire, c'est échapper au contrôle de l'autre, à son pouvoir, et dans cette réécriture-ci il a valeur de transgression puisqu'il est désobéissance à Dieu.

De fait, Sara se comporte comme une femme libre qui, tout au long du roman, pense et agit. La pensée d'abord: cette Sara romanesque bien différente de la Sarah biblique, questionne, doute, s'indigne, met en cause, juge, non sans finesse à en croire un narrateur qui lui semble tout acquis. À l'envers d'un homme docile devant Dieu, elle profère le «non» de ceux qui pensent: «Escucho y obedezco, respondió Abraham bajando la cabeza [...]. ¿No sabe nunca decir no este hombre blando como la cera de las colmenas?» (51).

Arrogance des émissaires divins que ses pensées critiques infantilisent ou condamnent, litiges avec le créateur même - «tiene declarado un pleito tenaz con el Mago» (46) — : Sara discrédite Dieu avec la même vigueur que l'Ėve insolente de Gioconda Belli: elle le dit capricieux, autoritaire, sinon malade de son pouvoir absolu, donneur d'ordres insensés et condamnant le rire tout en ordonnant de nommer leur fils Isaac, c'est-à-dire «celui qui rit» (203), injuste puisque châtiant coupables et innocents lors de la destruction terrible de Sodome et Gomorrhe, vengeur et faux lorsqu'il demande le sacrifice de leur fils - «jamás he visto a nadie más vengativo y rencoroso [...] rabió Sara, vengativo, rencoroso, falso, desleal y mentiroso» (220). Et comme Ėve revisitée par Gioconda Belli, cette Sara relue et "personnalisée" par Sergio Ramírez peut s'adresser au ToutPuissant au moyen d'impératifs qui inversent le rapport d'autorité: «déjanos en paz, ya es suficiente, búscate a otros, por qué nos persigues [...]» (19).

On observera que, tout au long du roman, les Écritures saintes sont relativisées par le jeu des versions discordantes et la contestation de pères de l'Église ou de théologiens qui font autorité, autant de façons de perturber opinions établies et conclusions admises depuis toujours, de dérouter le narrataire incité ainsi à

4 Entrevue à la librairie Lé à Madrid, 5 de marzo de 2015: https://www.youtube.com/ watch?v=NeK9aWQIV-I. Puis, blog d'un libraire: https://laslecturasdeguillermo. wordpress.com/2015/04/06/sara-de-sergio-ramirez/. 
repenser ce qu'il croyait acquis. La polyphonie, le multiperspectivisme, la vision subjective et parcellaire, confirment sur le plan formel l'impossibilité d'accéder à la vérité et, comme autant de réponses au dogme, invitent au doute et au respect de l'autre.

Sara n'éprouve pas uniquement sa liberté par la pensée critique, avons-nous dit, mais également par l'action. Plusieurs séquences en témoignent, notamment lorsqu'elle sauve Lot de la destruction de Sodome et Gomorrhe en agissant de façon ferme et stratégique, ou lorsqu'elle retient le bras d'Abraham sur le point de sacrifier leur fils, bondissant de sa cachette comme une panthère, dans une réécriture inattendue de l'épisode biblique. Dans les deux cas, Sara désobéit à un pouvoir abusif et mortifère - cet aspect constituant un fil rouge dans les romans de Sergio Ramírez -, dans les deux cas, elle est du côté de la vie et de la libération: "Ya he salido otras veces sin que te dieras cuenta, pensó Sara, siempre detrás de tus pasos para salvar a quien debe ser salvado» (233); c'est elle en définitive qui représente le salut face à un Dieu converti en pouvoir assassin.

Il faut préciser cependant qu'un personnage étrange appelé «el tuerto» influence Sara dans cette dernière séquence et qu'il apparaît comme un conspirateur à en juger par une sémantique qui politise son action; ce personnage inclassable qui connaît les décrets suprêmes du Mage, exhorte Sara à désobéir: "al inducirla a que interfiera en los planos trazados y los frustre, ha entrado en una conspiración en toda regla» (227), à quoi s'ajoutent sur la même page des termes convergents tels que "rebelión», "rebelde», "contrainteligencia», «exilio».

Pouvoir contesté parce que contestable, méprisable parfois, illusoire toujours, en définitive ridicule: on touche là un des enjeux de ces réécritures profanes et en un sens profanatrices, à savoir la démythification du pouvoir et, en regard de cette déconstruction, l'idéalisation avec la "construction" idéologique d'un monde meilleur dans lequel affleure, chez Gioconda Belli, le meilleur des mondes.

\section{Réécritures politiques: I'utopie et l'imagination au pouvoir?}

La réécriture de la Chute ne permet pas uniquement à Gioconda Belli de revenir sur la liberté de la femme, mais également sur le Paradis perdu, un aspect qui traverse son œuvre. El infinito en la palma de la mano, s'il est le récit de la Chute, s'achève non sur la perte mais sur l'espérance, la quête du Paradis, ou à tout le moins d'un paradis, comme un défi humain. Le Serpent explique à Ève que la nostalgie du Paradis peut devenir une formidable incitation à le retrouver, et l'invite à garder confiance dans ses facultés humaines pour en découvrir le chemin:

El Mal, el Bien, todo lo que es y será en este planeta se origina aquí mismo: en ti, en tus hijos, en las generaciones que vendrán. El conocimiento y la libertad son dones que tú, Eva, usaste por primera vez y que tus descendientes tendrán que aprender a utilizar por sí mismos. [...] La memoria del Paraíso nadará en su 
sangre y si logran comprender el juego de Elokim y no caer en las trampas que él mismo les tenderá, cerrarán los círculos del tiempo y reconocerán que el principio puede llegar a ser también el final. Para llegar allí nada tendrán sino la libertad y el conocimiento (201).

Dans les dernières lignes de ce roman, Ève exhorte Aklia, sœur jumelle d'Abel, à retrouver le Paradis tandis que celle-ci, muette après la mort de son frère, involue vers une forme d'innocence en rejoignant le monde simien - elle sera la mère de l'humanité. Le roman se clôt sur une pluie qui peut aussi bien symboliser la fertilité puisque la vie continue, que la purification avant que naisse un "nouveau" monde.

Mais quel monde l'homme est-il en mesure de créer? Gioconda Belli, qui dédie son livre aux victimes de la guerre d'Irak, a amplement expliqué, au moment de la parution de l'ouvrage, que lorsqu'elle écrivait ce roman elle lisait les informations sur cette zone géographique que la Bible désigne comme le berceau de l'humanité mais que l'homme a transformée en un monde apocalyptique et « cargada de ira y pena, luego había de imaginar el paraíso. Era una metáfora de la aspiración del sueño de la humanidad que nunca alcanzamos» (Geli 2008).

Dans cette réécriture mythique, tout à la fois poétique et réaliste, l'homme sur lequel réfléchit Gioconda Belli oscille constamment entre le Bien et le Mal, et demeure face à ce dilemme qu'il lui revient de résoudre. On trouve ce refus d'abdiquer dans d'autres écrits de Gioconda Belli qui font l'apologie du rêve ou dont l'intertextualité est lourde de sens, notamment lorsqu'elle emprunte au poète britannique lord Alfred Tennyson des vers épiques insérés dans son roman de l'utopie, Waslala: "To strive, to seek, to find and not to yield. Luchar, buscar, encontrar y nunca cejar» (1996: 136); ou lorsque, traduisant ceux de Thomas Stearns Eliot qui donnent à l'intention la primauté sur le résultat, elle célèbre:

El valor de la intención.

La lucha por recuperar lo que se ha perdido

lo que se perderá una y otra vez,

sin que, tal vez, se pierda o se gane.

La voluntad es lo que cuenta.

Lo demás no es asunto nuestro (1997: 65).

Dans Sara en revanche, nulle visée utopique n'oriente la critique du pouvoir que Sergio Ramírez oppose pourtant lui aussi à l'imagination: non pas celle qui conçoit le meilleur des mondes, mais celle qui fonde la littérature. Il n'a pas attendu Sara pour satiriser le pouvoir le plus souvent associé à l'animalité, c'est-à-dire un stade infra-humain, ou à l'abject, notamment au moyen du thème excrémentiel pour mieux rendre la souillure de la corruption, ou encore au monstrueux, sinon au démonique pour mieux le rapprocher du Mal. Sara ne déroge pas à la règle, qui déroule sous les yeux amusés du lecteur une galerie de portraits risibles.

Corps grotesques, scènes sexuelles pathétiques: le "corps du pouvoir" s'avère ridicule, qu'il s'agisse du pouvoir politique de Pharaon, dévirilisé - et dépossédé 
de son pouvoir? puisque affublé d'un petit phallus fripé (86) - et dont l'odeur répugnante signifie peut-être les miasmes du pouvoir; ou du roi Abimèlek ramené lui aussi à l'impuissance puisque empêché de toucher Sara par un Dieu le menaçant de mort (195); ou même du pouvoir divin représenté ici par des émissaires dont la plume de Sergio Ramírez exploite les traits angéliques sur le mode trivial: ainsi, Raphaël et Michaël, pas si différents physiquement des éphèbes les plus corrompus des lupanars selon Lot, déchaînent-ils les pulsions les plus primaires à Sodome et Gomorrhe où ils sont poursuivis par une foule lubrique (146).

Cette réécriture de versets bibliques, à plus d'un égard subversive ou transgressive, recourt à l'humour corrosif, à la "grotesquisation", la carnavalisation, comme à autant de schémas d'inversion. À l'image d'Aristophane moquant Socrate ou de Nietzsche qui se rit des maîtres qui ne se moquent pas d'euxmêmes dans Le gai savoir, Sergio Ramírez se rit du Mage en tant qu'il représente ici le pouvoir absolu et, comme tel, ne rit ni n'accepte que l'on rie. Baudelaire a produit à ce sujet une affirmation définitive dans «De l'essence du rire...»: «Le Sage par excellence, le Verbe Incarné, n’a jamais ri».

Mais si le rire est le propre de l'homme à en croire une fameuse assertion rabelaisienne, celui qui ne rit pas n'est-il pas “inhumain”?, le pouvoir n'estil pas inhumain? Ce pouvoir qui peut déshumaniser ceux qui le subissent, particulièrement dans les lettres latino-américaines, suppose également la déshumanisation de celui qui l'exerce, ce dont Sara témoigne amplement puisque plus d'une fois la protagoniste accuse l'inclémence des émissaires divins devenant anges exterminateurs à Sodome et Gomorrhe, tels les sbires sanguinaires d'un tout-puissant Mage qui rappelle ici les dictateurs les plus néroniens.

Ce pouvoir total et abusif, qui confine à la folie selon Sara n'en est pas moins insubstantiel fondamentalement si l'on en croit les confidences du Mage à son incorrigible Sara: «el cielo verdaderamente está vacío, todas esas estrellas del firmamento en realidad son falsas, murieron hace mucho tiempo aunque aún brillen [...] No es que sea falso, es que está muerto» (248) - il fait écho en cela à la comédie que Sara dénonce à plusieurs reprises. Grandeur et mensonge, grandeur et vacuité: on retrouve là une ligne de force des écrits de Sergio Ramírez qui associe presque systématiquement grandeur et décadence.

Pouvoir satirisé, pouvoir vidé de lui-même: on saisit l'amplitude critique de cette réécriture moins légère qu'il n'y semble, on en soupçonne la portée axiologique. Il n'est pas inédit que la littérature recoure au rire pour faire réfléchir; on sait que l'humour, qui présente la réalité sous un angle inattendu et révélateur, constitue un instrument critique efficace.

Quoiqu'avec bien plus de finesse que Gioconda Belli dont les écrits romanesques disent explicitement la volonté d'améliorer la société, une visée réformatrice n'est pas absente des romans de Sergio Ramírez qui, s'ils ne se veulent aucunement propagandistes, amènent à repenser le monde, à relativiser, 
notamment l'ordre établi, le pouvoir quel qu'il soit, ou toute idée se présentant sous les atours de la vérité. La parole est douteuse, rien n'est certain: aussi, les versions discordantes de ses récits révèlent-elles, outre l'impossibilité de connaître la vérité, une écriture antidogmatique.

Car si l'auteur a pu dire, en réécrivant l'histoire de Sarah: "No me meto con los dogmas, no los cuestiono" (Agüero 2015), il condamne en revanche «les canons inflexibles de l'orthodoxie religieuse» et répond à toute forme de rigidité par la tolérance, la capacité d'adopter la perspective de l'autre: «La mayor revolución es ver el mundo como lo ve el otro. En la política, pocos como Mandela o Luther King consiguieron encarnarse en el otro» (Bono 2015), a pu affirmer cet ancien révolutionnaire qui a assisté aux dérives autoritaires du gouvernement dont il était vice-président. Du roman Sara à la politique, et de la politique à l'écriture, l'auteur explique dans la même entrevue:

son antagónicas. El papel del escritor debe ser crítico; un escritor alienado sólo resulta una voz burocrática. Yo viví la experiencia en el poder. Defendía la causa como relacionista público de la revolución. Y realmente no podía ser crítico con lo que estaba viviendo. Hubiera sido un contrasentido. El espacio crítico es indisoluble de la escritura y el poder no te lo permite.

Dans une autre entrevue intitulée « La literatura está reñida con la militancia », l'écrivain revient sur cet aspect:

Yo recomiendo siempre a los alumnos que me encuentro en talleres y seminarios que no se metan en política, lo cual no quiere decir que no opinen. Uno tiene que tener una conciencia abierta y crítica, sobre todo en países con tantas anormalidades públicas como tienen los de América Latina. Pero la obra literaria debe abordarse desde la libertad y hablarle al poder, no plegarse a él; y eso es algo que, si uno pertenece a un partido, o forma parte de un régimen, se ve notablemente limitado (Gordo 2015).

Nous sommes face à des réécritures non dénuées d'une portée politique, soit qu'elles mettent en garde contre le pouvoir, soit qu'elles en appellent à un autre pouvoir ou un autre rapport au pouvoir, ce qui ne devrait pas nous surprendre de la part d'anciens révolutionnaires revenus des utopies sociales en partie seulement.

Gioconda Belli ne renonce pas à la quête d'un monde idéal, et Sergio Ramírez - qui a pu dire: "Je suis entré en politique parce que j'ai cru à son pouvoir transformateur» $(2000: 6)$ - poursuit son action, ou sa participation à ce monde, en imaginant, ce qui est une autre façon de rechercher l'utopie: "Imaginar, que es una forma de acercarse a la utopía. Al fin y al cabo, yo no he hecho a lo largo de mi vida sino imaginar. Imaginar mundos en mis libros, e imaginar un mundo mejor en mi vida. Oficios compartidos» (2000: 14).

Si l'imagination s'affiche ici contre le pouvoir, nous ne sommes pas loin, particulièrement avec Gioconda Belli, de "L'imagination au pouvoir» pour reprendre un slogan bien connu de Mai 68. Quoi qu'il en soit, ces deux auteurs peuvent réécrire, au nom des droits imprescriptibles de l'imagination pour lesquels plaidait Louis Aragon, des séquences bibliques avec le regard de leur 
temps, déconstruisant au besoin mythes et dogmes comme autant d'autorités consacrées, célébrant la liberté comme condition essentielle de notre humanité.

Quoique ces réécritures se veuillent humanistes et puissent être aisément universalisées, elles émanent d'une relecture toute personnelle des textes premiers. Quelle serait la nôtre? Et quelle (re)lecture faisons-nous de la leur? Notre lecture ne réécrit-elle pas leur texte à notre insu?, chaque lecture faisant l'objet de projections plus ou moins conscientes de la part du lecteur. À chacun sa relecture, et partant sa réécriture. Indéfiniment. La réécriture, intrinsèquement liée à l'écriture même, n'est donc pas près de s'abolir.

\section{Bibliographie}

Belli G., 2008, El infinito en la palma de la mano, Barcelona, Editorial Seix Barral. Belli G., 2001, El país bajo mi piel. Memorias de amor y guerra, Barcelona, Plaza \& Janés Editores.

Belli G., 1997, Apogeo, Managua, Anamá Ediciones Centroamericanas.

Belli G., 1996, Waslala, Emecé Editores, Barcelona.

Besse N., 2009, "El infinito en la palma de la mano de Gioconda Belli: el Hombre ante el Bien y el Mal», Carátula, Revista cultural centroamericana, n 30, junio-julio.

Besse N., 2010, «Les corps malmenés de Sergio Ramírez. Images d'un Nicaragua meurtri», Les représentations du corps dans la littérature latino-américaine, reCHERches $\mathrm{n}^{\circ} 4$ (ss dir. Nathalie Besse), Université de Strasbourg, p. 103-116.

Besse N., 2016, «La risa de una mujer frente al poder absoluto en Sara (2015) de Sergio Ramírez», Carátula, Revista cultural centroamericana, $\mathrm{n}^{\circ} 71$, abril.

Gérard A. M., 1989, Dictionnaire de la Bible, Paris, collection «Bouquins", Éditions Robert Laffont.

Kristeva J., 1969, Semeiotikè, Paris, Seuil.

Ramírez S., 2015, Sara, Madrid, Editorial Alfaguara.

Ramírez S., 2000, Oficios Compartidos/Un sandinismo en el que creer, CRLAArchivos, «Conferencias en el centro», Poitiers.

\section{Sitographie (entrevues)}

Agüero A., 2015, "Sara: una historia seductora», La Prensa Cultura, Managua, 12 juin: http://www.laprensa.com.ni/2015/06/12/cultura/1848609-sara-unahistoria-seductora

Bono F., 2015, «Sergio Ramírez: "La mayor revolución es ver el mundo como lo ve el otro" ", El País Cultura, Madrid, 5 mars: http://cultura.elpais.com/ cultura/2015/03/05/actualidad/1425584336_847976.html

Geli C., 2008, «Belli ganó con una novela sorprendente», El País, Barcelone, 6 février: http://www.elnuevodiario.com.ni/nacionales/7775-belli-ganonovela-sorprendente/ 
Gordo A., 2015, "Sergio Ramírez: "La literatura está reñida con la militancia” », $E l$ Cultural, Madrid, 6 mars: http://www.elcultural.com/noticias/letras/SergioRamirez-La-literatura-esta-renida-con-la-militancia/7493.

Mendoza A., 2015, "Sara” vista de otras maneras», La Prensa, Managua, 11 mars: http://www.laprensa.com.ni/2015/03/11/cultura/1796687-saravista-de-otras-maneras-se-inspira-en-su-nueva-novela-en-sara-y-abrahamdos-personajes-de-la-biblia.

Sevila Bolaños L., 2015, "Sergio Ramírez rompe el silencio de Sara», El Nuevo Diario, Managua, 14 juin: http://www.elnuevodiario.com.ni/suplementos/ cultural/362297-sergio-ramirez-rompe-silencio-sara-esposa-abraham/. 